\title{
Mycoplasma pneumoniae infection is associated with subacute cough
}

To the Editor:

Cough, one of the most common reasons patients seek medical attention [1], is categorised according to duration. Acute cough lasts $<3$ weeks, subacute cough lasts 3-8 weeks and chronic cough $>8$ weeks $[2,3]$. Acute cough is usually due to viral infection; it is transient and self-limiting [4]. Chronic cough has a variety of causes that can be established in $88-100 \%$ of patients using a diagnostic algorithm [3]. In contrast, the aetiology of subacute cough remains poorly defined, although post-infectious cough, sinusitis, post-nasal drip and bronchial asthma are listed as possible causes $[2,3,5,6]$. As subacute cough is often refractory to recommended therapies, a better understanding of its aetiology is needed. The present work examined a possible role for Mycoplasma pneumoniae infection in subacute cough.

Between July 2010 and June 2011, patients who visited the Dept of Respiration at the Affiliated Hospital of Academy of Military and Medical Sciences (AHAMMS, Beijing, China) on their own initiative were screened and enrolled if they met the following criteria: cough was the only major complaint; cough was not associated with haemoptysis; cough had lasted for 3-8 weeks (determined by patient recall); chest radiography was normal; and age $\geqslant 15$ years. Exclusion criteria included inpatient status, a prior history of lung or other systemic disease that could account for cough, current or past smoking, taking angiotensinconverting enzyme inhibitors, known immunodeficiency or pregnancy. Control subjects were enrolled from healthy medical staff in the department, their family members and relatives of patients who accompanied the patients. Control subjects had no cough for $\geqslant 1$ month and were subject to the same exclusion criteria as patients. Efforts were made to match controls with patients by age, sex and time of enrolment. The study was approved by the ethics committee of AHAMMS (protocol \#2010-08-99-1). Written informed consent was obtained from all patients and control subjects. Demographic data and medical history were documented using a pre-designed questionnaire. All symptoms and signs detected in a physical examination were recorded.

Oropharyngeal specimens were obtained from the posterior oropharynx using a sterile cotton swab from which M. pneumoniae was cultured [7]. A real-time quantitative PCR method was used to measure bacterial load by identifying $16 \mathrm{~S}$ rDNA of $M$. pneumoniae using the following primer and probe sequences: forward 5'-GCAAGGGTTCATTATTTG-3', reverse 5'-CGCCTGCGCTTGCTTTAC-3' and probe 5'-6carboxyfluorescein-AGGTAATGGCTAGAGTTTGACTG-tetramethylrhodamine-3'. PCR was performed in $25-\mu \mathrm{L}$ reactions containing $2 \times$ Premix Ex Taq (Takara, Dalian, China), $10 \mu \mathrm{M}$ of both primers, $3 \mu \mathrm{M}$ of the TaqMan probe and $3 \mu \mathrm{L}$ DNA template (prepared from swab agitate samples using QIAmp DNA mini kit (Qiagen China, Shanghai, China)) with a CFX96 Real-Time PCR Detection System (Bio-Rad, Hercules, CA, USA). PCR conditions were $93^{\circ} \mathrm{C}$ for $2 \mathrm{~min}, 10 \mathrm{cycles}$ at $93^{\circ} \mathrm{C}$ for $45 \mathrm{~s}$ and $55^{\circ} \mathrm{C}$ for $60 \mathrm{~s}$, followed by another 30 cycles at $93^{\circ} \mathrm{C}$ for $30 \mathrm{~s}$ and $55^{\circ} \mathrm{C}$ for $45 \mathrm{~s}$. Pure M. pneumoniae DNA (Da'an Gene Co Ltd, Guangzhou, China) was used to construct a standard curve for quantification.

To confirm the presence of M. pneumoniae, positive isolates, identified by both bacterial culture and realtime PCR, were subjected to nested PCR using two sets of primers directed against the organism-specific P1 gene sequence ( $P 1$ gene-based strain typing) [8] using $M$. pneumoniae strain FH (ATCC 15531) as a reference. PCR amplicons of the $P 1$ gene were characterised by agarose gel electrophoresis and nucleotide sequence determination.

Isolates identified as positive by both bacterial culture and PCR methods were tested for susceptibility to erythromycin, azithromycin, midecamycin, ofloxacin, levofloxacin, moxifloxacin, tetracycline, minocycline, amikacin and etimicin. Minimum inhibitory concentrations were determined by broth microdilution [7].

Patient and control groups were compared for the presence of M. pneumoniae using Fisher's exact test. Subgroup comparisons of clinical characteristics were performed using t-tests or the Chi-squared test (SPSS for Windows, version 17.0; IBM, Armonk, NY, USA). $\mathrm{p}<0.05$ was considered significant.

Out of 202 consecutive cough patients, 85 met the inclusion criteria. Two declined to participate. Excluded patients had the following illnesses: pneumonia $(n=50)$, post-flu/cold cough for $<2$ weeks $(n=31)$, interstitial lung disease $(n=15)$, bronchiectasis $(n=10)$, pulmonary hypersensitivity pneumonitis $(n=6)$ 
Patients

Subjects $\mathbf{n}$

M. pneumoniae detection

$\mathrm{Mp}^{+}$

$\mathrm{Mp}^{-}$

M. pneumoniae genotype

II

Ila

Age years

All subjects

$\mathrm{Mp}^{+}$

$\mathrm{Mp}^{-}$

Age group years

15-30

$\mathrm{Mp}^{+}$

$\mathrm{Mp}^{-}$

31-50

$\mathrm{Mp}^{+}$

$\mathrm{Mp}^{-}$

$\geqslant 51$

$\mathrm{Mp}^{+}$

$\mathrm{Mp}^{-}$

Sex

Male

$\mathrm{Mp}^{+}$

$\mathrm{Mp}^{-}$

Female

$\mathrm{Mp}^{+}$

$\mathrm{Mp}^{-}$

Season

Spring

$\mathrm{Mp}^{+}$

$\mathrm{Mp}^{-}$

Summer

$\mathrm{Mp}^{+}$

$\mathrm{Mp}^{-}$

Autumn

$\mathrm{Mp}^{+}$

$\mathrm{Mp}^{-}$

Winter

$\mathrm{Mp}^{+}$

$\mathrm{Mp}^{-}$
83

$46(55)$

37 (45)

$38(83)$

6 (13)

2 (4)

$37.3 \pm 13.2$

$38.4 \pm 14.1$

$35.7 \pm 12.0$

29 (35)

15 (33)

14 (38)

41 (49)

24 (52)

17 (46)

$13(16)$

7 (15)

$6(16)$

31 (37)

15 (33)

16 (43)

52 (63)

31 (67)

21 (57)

33 (40)

$14(30)$

19 (51)

8 (10)

1 (2)

7 (19)

16 (19)

10 (22)

6 (16)

26 (31)

21 (46)

5 (14)
Controls

p-value

80

4 (5)

76 (95)

3 (75)

1 (25)

0

$<0.0001$

0.715

0.615

$38.3 \pm 13.1$

$38.4 \pm 13.0$

$36.3 \pm 15.8$

26 (32)

2 (50)

24 (32)

39 (49)

1 (25)

38 (50)

15 (19)

1 (25)

14 (18)

$32(40)$

3 (75)

29 (38)

48 (60)

1 (25)

47 (62)

$30(38)$

1 (25)

29 (38)

$10(13)$

0

10 (13)

$20(25)$

0

$20(26)$

$20(25)$

$3(75)$

17 (22)

Data are presented as $\mathrm{n}(\%)$ or mean $+\mathrm{SD}$, unless otherwise stated. $\mathrm{Mp}^{+}:$M. pneumoniae-positive subjects; $\mathrm{Mp}^{-}$: M. pneumoniae-negative subjects.

and tuberculosis $(n=5) .24$ of the patients enrolled had prior consultations for cough; eight of them were prescribed a cephalosporin antibiotic for 3-5 days. 80 healthy controls were enrolled with a response rate of $100 \%$. No significant difference existed between patient and control groups with respect to age, sex or seasonal variation (table 1$)$.

Samples from 63 (76\%) out of 83 patients were culture-positive for M. pneumoniae, and 61 (74\%) out of 83 were found to be positive by real-time PCR (specificity $~ 80 \%) .46$ (55\%) samples from patients were positive by both culture and PCR methods, and all were confirmed as M. pneumoniae by P1 gene-based strain typing. In contrast, samples from four (5\%) out of 80 control subjects were culture-positive, and six (7.5\%) were PCR-positive for M. pneumoniae. All four positive isolates identified by both culture and PCR were confirmed as $M$. pneumoniae by $P 1$ gene-based strain typing. The $M$. pneumoniae detection frequency among subacute cough patients was significantly greater than among healthy controls ( $55 \%$ versus $5 \%$, $\mathrm{p}<0.0001)$. The difference would have been even more significant if either culture- or PCR-positive samples 
were counted as M. pneumoniae-positive samples ( $94 \%$ versus $7.5 \%, \mathrm{p}<0.0001)$, but such analysis would not exclude contribution from false-positives.

Quantitative real-time PCR revealed that 98\% (45 out of 46) M. pneumoniae-positive samples from the patient group had a bacterial DNA load of $10^{5}-10^{7}$ gene copies per $\mathrm{mL}$ of swab agitate suspension; $50 \%$ (23 out of 46 ) of these samples exhibited very high bacterial load $\left(\geqslant 10^{6}\right.$ gene copies per $\mathrm{mL}$ ). In contrast, the four M. pneumoniae-positive control samples gave only $10^{3}-10^{5}$ gene copies per $\mathrm{mL}$.

Of the 46 subacute cough patients identified as positive for M. pneumoniae by both culture and PCR methods, adolescents accounted for 33\% (15 out of 46), middle-aged adults 52\% (24 out of 46) and older adults $15 \%$ (seven out of 46 ). The 46 positive samples came from 15 males and 31 females. Seasonal effects were apparent: $21(46 \%)$ positive cases in the patient group were detected during the winter season, 14 $(30 \%)$ occurred in spring, $10(22 \%)$ in autumn, and one $(2 \%)$ in summer. Thus, infection with M. pneumoniae was significantly lower in summer $(\mathrm{p}=0.01)$.

Antimicrobial susceptibility data revealed that most isolates were resistant to macrolides and aminoglycosides. However, they were largely susceptible to tetracyclines and fluoroquinolones.

The present study showed, for the first time, that M. pneumoniae is highly prevalent in patients with subacute cough. Our conclusion is unlikely to be confounded by an M. pneumoniae outbreak, since an epidemiology study performed in the same area and overlapping most of our study duration revealed only $18 \%$ infection frequency among 500 community-acquired pneumonia patients [9]. Almost all samples from patients exhibited high $M$. pneumoniae DNA load ( $\geqslant 10^{5} 16 \mathrm{~S}$ gene copies per $\mathrm{mL}$ ). Among the factors analysed, season is the only risk factor that significantly affected $M$. pneumoniae detection rate; prevalence was significantly lower in summer $(p=0.01)$. Antimicrobial susceptibility assays indicated that most M. pneumoniae isolates were nonsusceptible to both macrolides and aminoglycosides but highly susceptible to tetracyclines and fluoroquinolones. Thus, M. pneumoniae infection may play a significant role in subacute cough, at least among Chinese populations in the Beijing area. Although the American College of Chest Physicians evidence-based clinical practice guidelines assert no role for antibiotic therapy [10], because subacute cough is not thought to arise from bacterial infection, our work encourages the investigation of treatment benefit with tetracyclines or fluoroquinolones.

0

@ERSpublications

Subacute cough is associated with the presence of Mycoplasma pneumoniae, thus antimicrobial therapy may be beneficial http://ow.ly/sPAsX

Xin Yuan ${ }^{1,2,7}$, Youning Liu ${ }^{1,7}$, Changqing $\mathrm{Bai}^{2}$, Yuan $\mathrm{Luo}^{3}$, Rui Wang ${ }^{4}$, Rentao Wang ${ }^{1}$, Yun Cai ${ }^{4}$ and Xilin Zhao ${ }^{1,5,6}$ ${ }^{1}$ Dept of Respiration, PLA General Hospital, Beijing, ${ }^{2}$ Dept of Respiration, Affiliated Hospital of Academy of Military Medical Sciences, Beijing, ${ }^{3}$ Institutes of Pharmacology and Toxicology, Academy of Military Medical Sciences, Beijing, ${ }^{4}$ Dept of Clinical Pharmacology, PLA General Hospital, Beijing, and ${ }^{5}$ State Key Laboratory of Molecular Vaccinology and Molecular Diagnostics, School of Public Health, Xiamen University, Xiamen, China. ${ }^{6}$ Public Health Research Institute Center and Dept of Microbiology and Molecular Genetics, New Jersey Medical School, Rutgers Biomedical and Health Sciences, Newark, NJ, USA. ${ }^{7}$ Both authors contributed equally.

Correspondence: X. Zhao, Public Health Research Institute Center and Dept of Microbiology and Molecular Genetics, New Jersey Medical School, Rutgers Biomedical and Health Sciences, 225 Warren Street, Room W310F, Newark, NJ 07103, USA. E-mail: zhaox5@njms.rutgers.edu

Received: April 162013 | Accepted after revision: Oct 312013 | First published online: Nov 142013

Support statement: This study was supported by a grant from the National Natural Science Foundation of China (grant no. 30670927).

Conflict of interest: Disclosures can be found alongside the online version of this article at www.erj.ersjournals.com

Acknowledgements: We would like to thank the enrolled subjects of the study and Y. Li, W-K. Niu, X.Y. Shang, Z.Z. Heng, Y.H. Liu, Y. Liang and Q. Liu (Dept of Respiration, Affiliated Hospital of Academy of Military Medical Sciences, Beijing, China) for their help with the collection of specimens and clinical data. We also thank K. Drlica (Public Health Research Institute, New Jersey Medical School, Rutgers Biomedical and Health Sciences, Newark, NJ, USA) for his critical comments on the manuscript.

\section{References}

Cherry DK, Hing E, Woodwell DA, et al. National Ambulatory Medical Care Survey: 2006 summary. Natl Health Stat Report 2008; 3: 1-39.

2 Irwin RS, Baumann MH, Bolser DC, et al. Diagnosis and management of cough executive summary: ACCP evidence-based clinical practice guidelines. Chest 2006; 129: Suppl. 1, 1S-23S.

3 Irwin RS, Madison JM. The diagnosis and treatment of cough. N Engl J Med 2000; 343: 1715-1721. 
4 Pratter MR. Cough and the common cold: ACCP evidence-based clinical practice guidelines. Chest 2006; 129: Suppl. 1, 72S-74S.

5 Poe RH, Harder RV, Israel RH, et al. Chronic persistent cough. Experience in diagnosis and outcome using an anatomic diagnostic protocol. Chest 1989; 95: 723-728.

Kwon NH, Oh MJ, Min TH, et al. Causes and clinical features of subacute cough. Chest 2006; 129: $1142-1147$.

Waites K, Bébéar C, Robertson JA, et al. Laboratory Diagnosis of Mycoplasma Infections. Cumitech 34. Washington DC, American Society for Microbiology, 2001.

8 Kong F, Gordon S, Gilbert GL. Rapid-cycle PCR for detection and typing of Mycoplasma pneumoniae in clinical specimens. J Clin Microbiol 2000; 38: 4256-4259.

9 Liu YF, Gao Y, Chen MF, et al. Etiological analysis and predictive diagnostic model building of communityacquired pneumonia in adult outpatients in Beijing, China. BMC Infect Dis 2013; 13: 309.

10 Braman SS. Postinfectious cough: ACCP evidence-based clinical practice guidelines. Chest 2006; 129: Suppl. 1, 138S-146S.

\section{Maternal 25-hydroxyvitamin D levels in relation to offspring respiratory symptoms and infections}

\section{To the Editor:}

Recently, there has been an increasing interest in the immunomodulatory effects of vitamin D. Several studies have suggested detrimental effects of insufficient 25-hydroxyvitamin D (25(OH)D) levels on the innate and acquired immune system, which may contribute to the development of infections and atopic and allergic conditions [1-4]. Children and pregnant and lactating females have been identified as groups with a high risk of 25(OH)D insufficiency [5]. Low maternal serum 25(OH)D levels in pregnancy may contribute to increased risk of infections and atopic outcomes in offspring. Previous studies demonstrate inconsistency regarding relationships between maternal vitamin D intake, serum 25(OH)D levels and umbilical cord $25(\mathrm{OH}) \mathrm{D}$ levels with these outcomes in offspring [6-10]. We aimed to explore relationships between maternal serum $25(\mathrm{OH}) \mathrm{D}$ levels during late pregnancy and parent-reported respiratory tract symptoms and doctor-diagnosed lower respiratory tract infections (LRTI) in early childhood in a large cohort study.

The study sample consisted of 2025 mother-child pairs from the Southampton Women's Survey with maternal serum 25(OH)D measurement at 34 weeks' gestation (DiaSorin radioimmunoassay; Diasorin, Stillwater, MN, USA) [11]. Follow-up was at children's age 6 months $(\mathrm{n}=2025), 12$ months $(\mathrm{n}=1946)$ and 2 years $(n=1876)$. Parents were asked whether the child had suffered from any of the following since the last visit: one or more episodes of chest wheezing/whistling, waking at night coughing for three or more nights in a row (prolonged cough), one or more episodes of croup or a croupy cough, bouts of vomiting or diarrhoea $\geqslant 2$ days, or a doctor-diagnosed chest infection, bronchitis, bronchiolitis, pneumonia and/or ear infection. Chest infection, bronchitis, bronchiolitis and pneumonia were combined into one variable labelled "LRTI". Binary variables were created for each outcome. Relative risks were calculated using Poisson regression with robust variance with serum $25(\mathrm{OH}) \mathrm{D}>75 \mathrm{nmol} \cdot \mathrm{L}^{-1}$ as the reference category [12]. All analyses were adjusted for child's sex, birthweight and gestational age, and for maternal age at childbirth, educational level, prepregnancy body mass index, parity, ethnicity, smoking in pregnancy and duration of breastfeeding. The study was approved by the Southampton and South West Hampshire Research Ethics Committee (276/97, 307/97, 089/99 and 06/Q1702/104). Consent was obtained before the inclusion of participants.

Median (interquartile range) late-pregnancy serum 25(OH)D level was $59.0(40.6-84.3) \mathrm{nmol} \cdot \mathrm{L}^{-1}$. Lower latepregnancy serum $25(\mathrm{OH}) \mathrm{D}$ levels were not associated with increased risk of parent-reported respiratory symptoms or infections in children aged 6 months, 12 months or 2 years. On the contrary, mothers with serum $25(\mathrm{OH}) \mathrm{D}$ levels $<50 \mathrm{nmol} \cdot \mathrm{L}^{-1}$ reported fewer respiratory symptoms and doctor-diagnosed LRTI in their children aged 0-6 months than those with serum 25(OH)D levels $>75 \mathrm{nmol} \cdot \mathrm{L}^{-1}$ (table 1). Additional adjustment for season of blood sampling (April to September versus October to March) did not alter our findings.

Our results do not support an association between low late-pregnancy serum 25(OH)D levels and increased risk of parent-reported offspring respiratory symptoms and infections in early childhood. The positive associations between serum 25(OH)D levels and self-reported respiratory symptoms and LRTI at 0-6 months may be attributable to residual confounding. Thus, health conscious females have higher serum 